\title{
Tensiones en uniones soldadas a tope con perfiles abiertos bajo torsión
}

\author{
Stress in butt welded joints of open profiles under torsion \\ Hernán Hernández Herrera ${ }^{1}$ Rafael Goytisolo Espinosa ${ }^{2}$ \\ Juan José Cabello Eras ${ }^{1} \quad$ Milen Balbis Morejón ${ }^{1}$ \\ Recibido 29 de mayo de 2014, aceptado 5 de marzo de 2015 \\ Received: May 29, 2014 Accepted: March 5, 2015
}

\begin{abstract}
RESUMEN
En el trabajo se destaca como en la literatura de Mecánica de Materiales y Diseño de Elementos de Máquinas, solo algunos autores abordan el tema de las uniones soldadas, dentro de estos se pueden citar a Shigley, Hall, Reshetov y Faires [1, 3, 4-5]; estos autores, aunque brindan expresiones para el cálculo de las tensiones en uniones soldadas de configuración geométrica compleja, no analizan el cálculo de las mismas para el caso de estar sometidas a torsión, tampoco toman en cuenta para los cálculos las particularidades de los perfiles de paredes delgadas, esto solo es analizado en el Tratado general de soldadura de los autores Schimpke, Horn y Ruge [7], donde se mencionan estas particularidades sin abordar las expresiones para el cálculo de las tensiones en casos complejos. Un tanto similar ocurre en los artículos y literatura especializada, donde se profundiza en la investigación sobre los parámetros tecnológicos en la soldadura, materiales y otros temas sin abordar el perfeccionamiento de las expresiones de cálculo para determinar las tensiones en este tipo de uniones. En el presente trabajo se brindan nuevas expresiones para el cálculo de las tensiones tangenciales que surgen en los cordones de uniones a tope, en perfiles abiertos sometidos a torsión libre, tomando en cuenta para ello su consideración como perfiles de paredes delgadas.
\end{abstract}

Palabras clave: Uniones soldadas, tensiones, torsión, perfiles abiertos, perfiles de paredes delgadas.

\begin{abstract}
In this paper is addressed how in the literature of Mechanic of Materials and Machine Elements Design only few authors address the subject of welded joints, among those can be mentioned Shigley Hall, Reshetov, and Faires [1, 3, 4-5]; although they provide expressions to calculate stress in complex geometric configurations of welded joints, they do not analyze calculation for butt welded joints subject to torsion, neither take into account characteristics of thin-walled side faces for the calculations. Only in the General Treaty of Schimpke Welding, Horn and Ruge [7] are mentioned these features without addressing the expressions for the calculation of stresses in cases of complex cases. Similar case is seen in articles and specialized literature wheredelves into research on the technological parameters in welding, materials and other subjects without addressing the improvement of calculation expressions to determine the stresses in such joints. In this paper new expressions are shown for the calculation of tangential stresses arising on the welds on butt joints of open profiles under free torsion, taking into account thin-wall side faces.
\end{abstract}

Keywords: Welded joints, stress, torsion, open profiles, profiles thin walls.

\footnotetext{
1 Grupo de Investigación en Optimización Energética GIOPEN. Facultad de Ingeniería. Universidad de la Costa CUC. Calle 58 \#55-66. CP 080006. Barranquilla, Colombia. E-mail: hhernand16@cuc.edu.co; mbalbis1@ cuc.edu.co; jcabello@cuc.edu.co

2 Facultad de Ingeniería Mecánica. Universidad de Cienfuegos. Carretera a Rodas Km 4, Cuatro Caminos. Cienfuegos. Cuba. CP 59430. E-mail: ragoyti@ucf.edu.cu
} 


\section{INTRODUCCIÓN}

La soldadura es la unión permanente más utilizada en la construcción de maquinaria, por lo que resulta sorprendente que en la literatura especializada no se trate con frecuencia ni con suficiente profundidad y especificidad la evaluación de su resistencia, lo que fue destacado por Shigley "Una de las dificultades que afronta el ingeniero en el diseño al trabajar con uniones soldadas, es que a esta materia no se le ha dedicado el mismo estudio riguroso que a otros procesos, materiales y elementos mecánicos" [1]. En el diseño de uniones soldadas es frecuente la introducción de factores de seguridad elevados y la existencia de códigos preestablecidos para el diseño de uniones en equipos, como los recipientes que trabajan a presión y otros de destino específico con altos requisitos de seguridad en su construcción y operación.

La Especificación para Construcciones de Acero ANSI-AISC 360-10 [2] establece las relaciones geométricas que deben tener los elementos de refuerzos para las uniones mixtas bajo diferentes solicitudes de carga, así como los requerimientos técnicos que deben cumplir estos refuerzos y los métodos de soldadura más adecuados a utilizar en función de la solicitación a que están sometidos. Se analizan cada uno de estos métodos mostrándose el área efectiva para estas uniones, las limitaciones de cada uno y las dimensiones mínimas de los cordones de soldadura para cada caso.

En la literatura especializada en el cálculo y diseño de Elementos de Máquinas [1, 3-5] se aborda el tema de la resistencia de las costuras soldadas de manera general, enunciando sus principios generales y las solicitaciones más frecuentes a que son sometidas, además resumen en tablas las características geométricas fundamentales de las costuras más usuales y exponen herramientas para el cálculo de cordones simples a partir de la solución de ejemplos típicos. Sin embargo es de destacar que el caso de la torsión se aborda muy someramente y en particular el caso de cordones de soldadura a tope, bajo esta solicitación, se obvia por completo.

En la literatura sobre cálculo y diseño de estructuras metálicas se profundiza más en el tema, Mukanov [6] aborda profundamente los aspectos constructivos de estas uniones y trata incluso el cálculo a fatiga de las mismas, pero solo aborda los casos más simples de carga, obviando casos específicos como los cordones entre vigas de diversos perfiles sometidos a torsión.

Schimpke, Horn y Ruge [7] en su Tratado general de soldadura mencionan la similitud de los cordones soldados entre vigas con secciones transversales complejas y los perfiles de vigas de paredes delgadas, sin embargo no la aplican al desarrollo de las ecuaciones para evaluar la resistencia de uniones soldadas con perfiles complejos.

La investigación desarrollada por Hernández [8] se orienta al estudio de las uniones soldadas con costuras de filete analizándolas como un perfil de paredes delgadas, donde se obtienen nuevas expresiones para el cálculo de las tensiones tangenciales que se producen cuando estos cordones son sometidos a torsión.

En las revistas especializadas en soldadura, varios autores abordan los problemas relativos a la elevación de la resistencia en las uniones soldadas, mediante el mejoramiento y control de los parámetros tecnológicos, su ejecución o los materiales de soldadura [9-11]. Otros se enfocan en la importancia que tienen las tensiones residuales producidas por los efectos de la temperatura, presentes en casi todas las uniones soldadas, y la influencia que tienen estas en el agrietamiento y la destrucción de las mismas [12-17], también se estudia la evaluación de la resistencia de las costuras soldadas aplicando los métodos de la Mecánica de la Fractura [18-19], la determinación de los factores de concentración de tensiones en la zona de la unión [20-21] y el cálculo a fatiga de las uniones soldadas [22].

Urbuan [23] aborda el tema de las uniones soldadas para la fabricación de estructuras metálicas, analiza los principales aspectos que afectan su durabilidad, los diseños recomendados para evitarlos, los métodos de soldaduras, tipos de uniones y cordones, posibles defectos, así como diferentes disposiciones constructivas para estructuras metálicas.

En la investigación desarrollada por Fraile [24] se abordan casos específicos de uniones entre vigas de alma llena, "perfil I-H" y "perfil doble T", las que se utilizan fundamentalmente en aplicaciones sometidas a flexión, lo que se debe a su baja resistencia a la torsión. En el trabajo se propone una metodología 
que permite diseñar los cordones para solicitaciones especificadas y se muestran varios ejemplos, sin embargo no se abordan los cordones de soldadura sometidos a torsión.

Aunque en la literatura se exponen los principios del cálculo de las configuraciones y solicitaciones más típicas en uniones soldadas, resulta evidente la necesidad de contar con métodos de cálculo específicos para las configuraciones menos usuales que les permitan a los ingenieros evaluar su resistencia y diseñarlas de forma racional, este es el caso de las uniones soldadas de vigas de perfiles complejos soldadas a tope y sometidas a torsión, las que se utilizan fundamentalmente en la fabricación de estructuras soldadas para diversos fines y para los cuales en la literatura no se registran los métodos y ecuaciones para su diseño. Por ello el presente trabajo tiene como objetivo la obtención de ecuaciones que permitan abordar el cálculo de resistencia de uniones soldadas mediante cordones a tope de configuración compleja sometidos a torsión considerando los mismos como perfiles de paredes delgadas.

\section{METODOLOGÍA}

Para la evaluación de la resistencia de las uniones soldadas con cordón a tope, sometidas a torsión, se utiliza la ecuación clásica de la mecánica de materiales que permite determinar las tensiones tangenciales máximas que se producen en la sección transversal del cordón y compararlas con su tensión de cortante admisible:

$$
\tau_{M t}=\frac{M t}{W t} \leq[\tau]
$$

Donde:

$\tau_{M t}$-Tensión tangencial máxima en el cordón de soldadura, MPa.

Mt-Momento de torsión que actúa sobre el cordón de soldadura, $\mathrm{N}-\mathrm{mm}$.

Wt- Parámetro geométrico de la sección sometida a torsión, $\mathrm{mm}^{3}$.

$[\tau]$ - Tensión admisible del material del cordón de soldadura. MPa.

El uso de esta ecuación es perfectamente válido y preciso cuando se trata de cordones con geometría simple, como perfiles circulares, rectangulares, cuadrados o triangulares, bien estudiados en los que la distribución de las tensiones tangenciales es comprendida y en la literatura se dispone de las ecuaciones necesarias para la determinación de sus valores máximos y de la zona donde estas actúan.

Sin embargo, cuando la configuración del cordón de soldadura adquiere formas más complejas, como los perfiles mostrados en la Figura 1, esta ecuación introduce imprecisiones tanto en la determinación de las tensiones máximas como de la zona donde actúan.
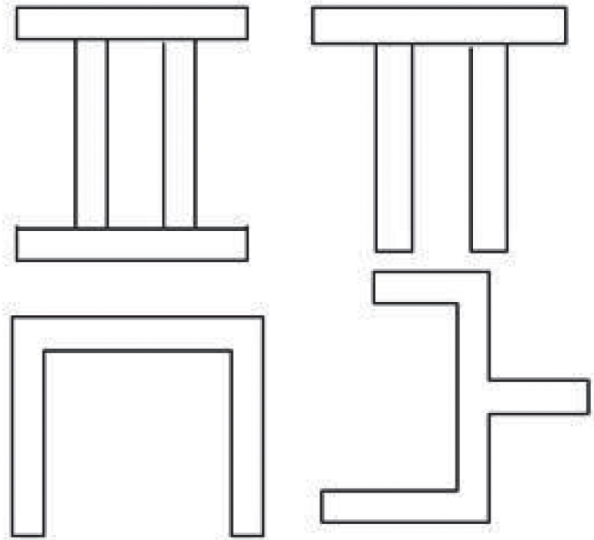

Figura 1. Cordones de soldadura con perfiles complejos.

En la Figura 1 se puede apreciar que estos perfiles cumplen la condición geométrica establecida para considerarlos como una viga de paredes delgadas según su sección, esta condición consiste en que su perímetro sea mucho mayor que el espesor.

El punto de partida del presente trabajo es la aplicación del aparato matemático desarrollado para el cálculo de las tensiones en vigas de paredes delgadas a torsión, al cálculo de las tensiones en cordones de soldadura a tope sometidos a torsión, cuya configuración permita considerarlos como un perfil de paredes delgadas.

Los perfiles de paredes delgadas se dividen en abiertos y cerrados. El carácter de la distribución de las tensiones tangenciales a través del espesor de la pared es diferente en ellos y se establece utilizando la analogía de la membrana. 
Esta analogía consiste en considerar que la distribución de tensiones en el perfil de paredes delgadas sometido a torsión es similar al de las tensiones que se producen en una membrana, extendida sobre una placa plana, con un agujero semejante al perfil analizado y sometida a una presión normal.

En la Figura 2 se muestra el comportamiento de la membrana para un perfil abierto, en el mismo su deformación es curva y la tensión se determina como la pendiente de esta variable siendo máximas en los extremos y mínimas en el centro del perfil como se puede apreciar en la figura.

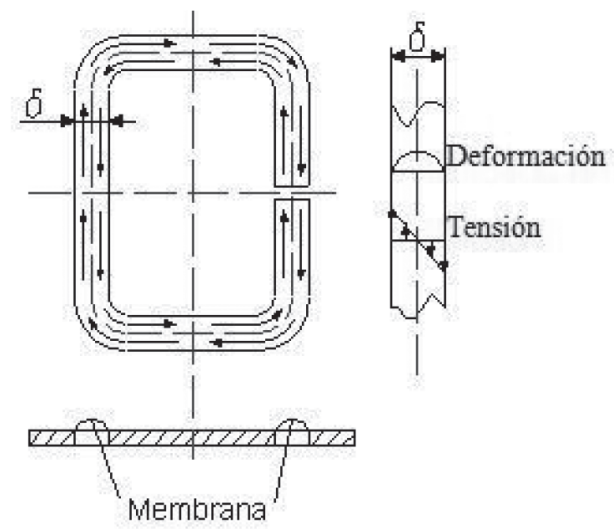

Figura 2. Analogía de la membrana para un perfil de paredes delgadas abierto.

En la Figura 3 se muestra el comportamiento en un perfil cerrado, en el que la deformación de la membrana será lineal y la distribución de la tensión en el espesor del perfil es constante.

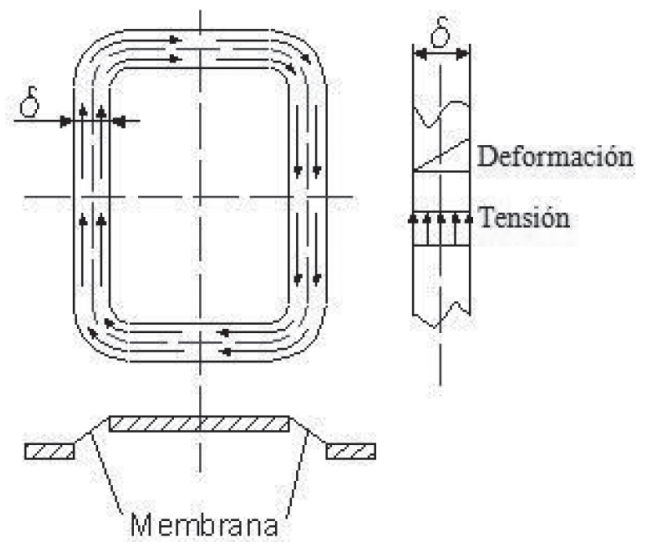

Figura 3. Analogía de la membrana para un perfil de paredes delgadas cerrado.
El cálculo de las tensiones en el caso de los perfiles abiertos se fundamenta en el hecho de que la magnitud y distribución de las mismas en la sección no se modifican significativamente si se endereza el perfil como se muestra en la Figura 4 siendo $S_{4}$ la suma de $S_{1}+S_{2}+S_{3}$. Es decir, las tensiones en un perfil abierto de configuración quebrado serán aproximadamente las mismas que uno de perfil rectilíneo. En este caso, uno de los lados es el perímetro "S" y el otro es el espesor" $\delta$ ". Como: $S / \delta>10$, utilizando la expresión para una sección rectangular se tiene que $\alpha=\beta$ $=0,333[4,6]$ y la tensión tangencial máxima se calcula como:

$$
\tau_{\max }=\frac{M_{t}}{W_{t}}
$$

Donde:

$$
W_{t}=\frac{\delta^{2} \cdot s}{3}
$$
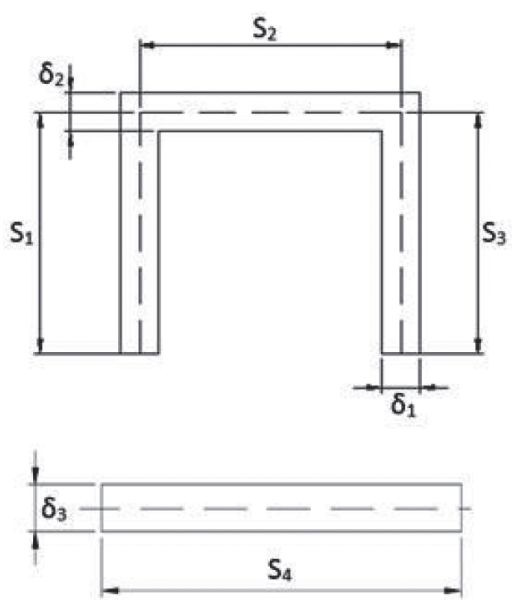

Figura 4. Perfil abierto desarrollable.

Cuando se trata de un perfil de paredes delgadas abierto y compuesto que no puede ser enderezado y transformado en un rectángulo, como el mostrado en la Figura 5, según la analogía de la membrana se establece que la mayor tensión se produce en el tramo de mayor espesor $\delta_{\text {máx }}$ donde $\tau_{\text {máx }}$ se produce para $\delta_{\mathrm{i}}=\delta_{\text {máx }}$, siendo la ecuación para el cálculo de las tensiones:

$$
\tau_{\max }=\frac{M_{t}}{W_{t}}=\frac{3 \cdot M_{t} \cdot \delta_{\max }}{\delta_{1}^{3} s_{1}+\delta_{2}^{3} s_{2}+\ldots+\delta_{n}^{3} s_{n}}
$$


Donde:

$$
W_{t}=\frac{I_{t}}{\delta_{\max }}=\frac{\delta_{1}^{3} s_{1}+\delta_{2}^{3} s_{2}+\ldots+\delta_{n}^{3} s_{n}}{3 \cdot \delta_{\max }}
$$

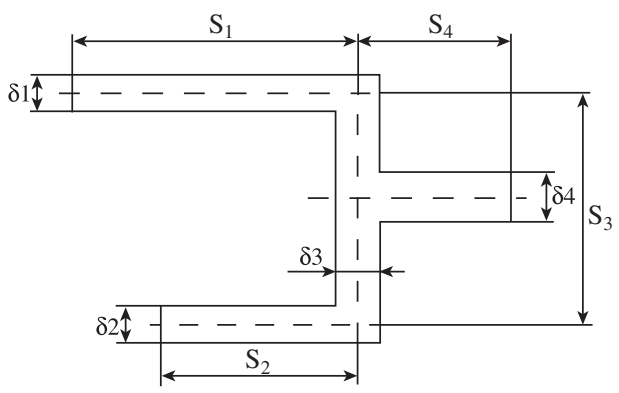

Figura 5. Perfil de paredes delgadas abierto no desarrollable.

En el presente trabajo se aplican las expresiones descritas anteriormente, para realizar el cálculo de las tensiones en cordones de soldadura con perfiles abiertos de paredes delgadas, y se obtienen nuevas expresiones que permiten calcular las tensiones tangenciales máximas en tres diferentes uniones con soldadura continua a tope y sometidas a torsión pura.

Las expresiones obtenidas para estas tres uniones se muestran en la Tabla 1. Las mismas son validadas por medio de tres ejemplos en los que se determinan analíticamente los valores de las tensiones y posteriormente se modelan por el Método de los Elementos Finitos utilizando el programa Cosmos Design para comparar los resultados.

La modelación por el Método de los Elementos Finitos parte de un análisis estático donde se tienen en cuenta las siguientes consideraciones:

- el material que se utiliza es el (AISI 1020) un acero de bajo contenido de carbono muy común en estructuras soldadas.

- en cada uno de los modelos elaborados se realizó el estudio de convergencia correspondiente, afinando la malla progresivamente hasta obtener una diferencia entre los valores de las tensiones para el modelo definitivo y el modelo precedente nunca superior a un 5\%, y de esta manera garantizar que los valores de las tensiones máximas locales no dependieran del tamaño de la malla utilizada, las mismas en todos los casos contenían más de 30000 nodos.

- para la realización del mallado se utiliza la malla de mayor calidad utilizando el elemento tetraédrico parabólico, el que brinda adecuados resultados por tener mayores posibilidades de generar la configuración del contorno.

- para garantizar que la sección estuviera sometida a cortante puro en cada extremo de las secciones se aplica un momento torsor para así evitar la aparición de la torsión restringida y el efecto del bimomento que sufre el perfil.

En la Figura 6 se muestran las diferentes uniones analizadas, estas se encuentran soldadas en su sección media y sometidas a cortante puro mediante un momento torsor en ambos extremos con un valor de $\mathrm{Mt}=800 \mathrm{kN}-\mathrm{mm}$, también se muestran las dimensiones de cada una de ellas.
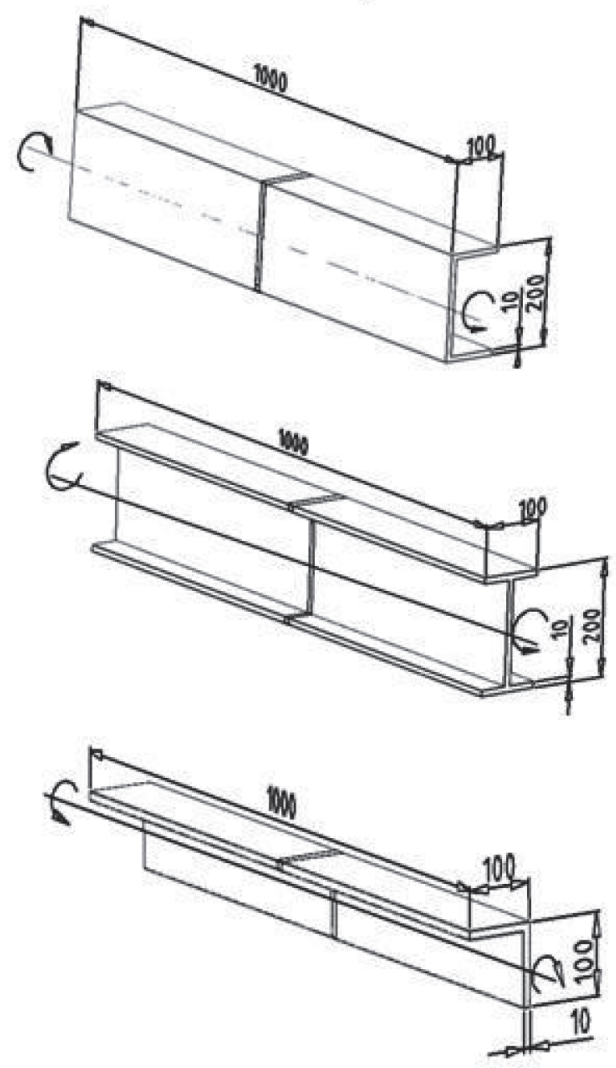

Figura 6. Uniones soldadas analizadas. 


\section{RESULTADOS}

A continuación se muestran los resultados obtenidos analíticamente para las diferentes uniones soldadas mostradas en la Figura 6, utilizando las expresiones brindadas en la Tabla 1.

Para la unión en U

$\mathrm{L}_{1}=\mathrm{L}_{2}=95 \mathrm{~mm} \mathrm{~L}_{3}=190 \mathrm{~mm} \mathrm{~S}=10 \mathrm{~mm}$

$$
\begin{aligned}
\tau_{M t} & =\frac{3 \cdot M t}{s^{2}\left(L_{1}+L_{2}+L_{3}\right)} \\
\tau_{M t} & =\frac{3 \cdot 800 \mathrm{kN} \cdot \mathrm{mm}}{(10 \mathrm{~mm})^{2}(2 \cdot 95 \mathrm{~mm}+190 \mathrm{~mm})}=63,15 \mathrm{MPa}
\end{aligned}
$$

Para la unión I

$\mathrm{S}_{1}=\mathrm{S}_{2}=\mathrm{S}_{3}=10 \mathrm{~mm} ; \mathrm{L}_{2}=\mathrm{L}_{3}=100 \mathrm{~mm}$ y $\mathrm{L}_{1}=190 \mathrm{~mm}$

$$
\tau_{\text {máx }}=\frac{3 \cdot M t \cdot \delta_{\max }}{\delta^{3}\left(L_{1}+L_{2}+L_{3}\right)}
$$

Como $\delta_{\text {máx }}=\delta$

$$
\begin{aligned}
\tau_{\text {máx }} & =\frac{3 \cdot M t}{\delta^{2}\left(L_{1}+L_{2}+L_{3}\right)} \\
\tau_{\text {máx }} & =\frac{3 \cdot 800 \mathrm{kN} \cdot \mathrm{mm}}{(10 \mathrm{~mm})^{2}(2 \cdot 100 \mathrm{~mm}+190 \mathrm{~mm})}=63,53 \mathrm{MPa}
\end{aligned}
$$

Para la unión L

Teniendo en cuenta que en este caso: $\mathrm{S}_{1}=\mathrm{S}_{2}=10$ $\mathrm{mm} ; \mathrm{l}_{1}=\mathrm{l}_{2}=100 \mathrm{~mm}$

$$
\begin{aligned}
\tau_{M t} & =\frac{3 \cdot M t}{s^{2}\left(l_{1}+l_{2}\right)} \\
\tau_{M t} & =\frac{3 \cdot 800 \mathrm{kN} \cdot \mathrm{mm}}{10 \mathrm{~mm}^{2}(100 \mathrm{~mm}+100 \mathrm{~mm})}=120 \mathrm{MPa}
\end{aligned}
$$

En la Figura 7 se muestra el valor de la tensión equivalente según la 4ta Hipótesis de Resistencia de Von Mises, obtenida para cada una de las uniones, producto del procesamiento de las mismas por medio del Método de los Elementos Finitos utilizando el programa Cosmos Design. Para todos los casos se muestra el valor de las tensiones en el plano medio de la unión soldada.

Para el caso de la Viga I la tensión máxima alcanza una magnitud de $\sigma_{\text {eq }}=133 \mathrm{MPa}$ según el criterio de Von Mises. La Tensión tangencial máxima según este von Mises (N/mm² (MPa))
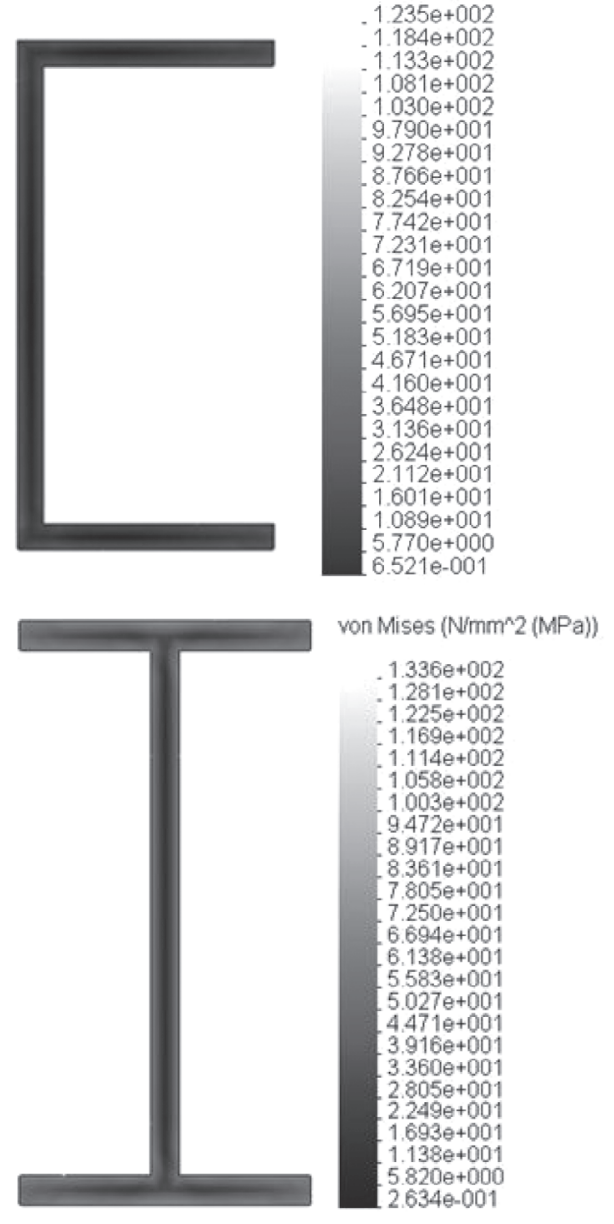

von Mises (N/mm² (MPa))
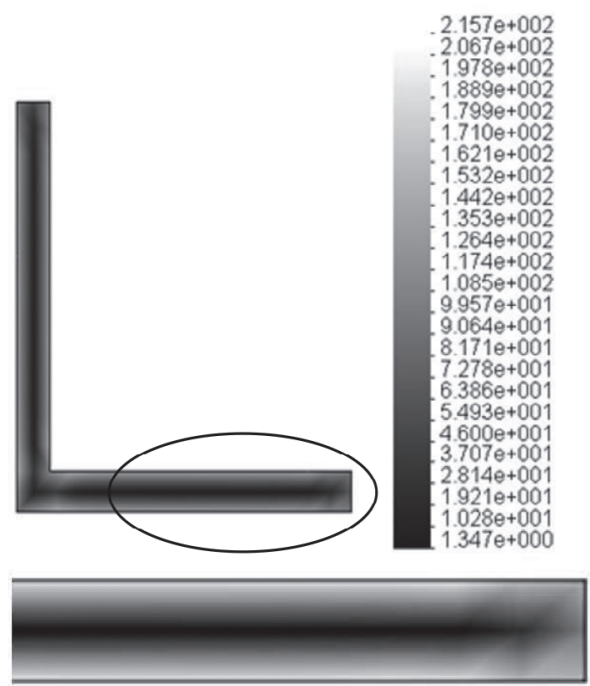

Figura 7. Tensiones obtenidas por el MEF para las diferentes uniones soldadas. 
propio criterio de Von Mises, al no existir tensiones normales, se puede calcular por la expresión: $\tau_{\text {máx }}=\frac{\sigma_{e q}}{\sqrt{3}}=76,71 \mathrm{MPa}$; en el caso del perfil $\mathrm{U}$ el valor de la tensión máxima es de $\sigma_{\text {eq }}=123 \mathrm{MPa}$ siendo $\tau_{\text {máx }}=71,01 \mathrm{MPa}$ y para el perfil en L el valor es de $\sigma_{\text {eq }}=215 \mathrm{MPa}$ y $\tau_{\text {máx }}=124,1 \mathrm{MPa}$.
Para todos los casos los valores de las tensiones obtenidas por el MEF están en correspondencia con las obtenidas analíticamente y se puede apreciar que los valores de las tensiones máximas aparecen en los contornos de las mismas y son uniformes a lo largo de estos, siendo mínimos en el centro del perfil como se expresa en la analogía de la membrana para el caso de los perfiles abiertos, lo que demuestra

Tabla 1. Expresiones para el cálculo de las tensiones tangenciales máximas en las uniones soldadas a tope analizadas, con perfiles abiertos de cateto constante, de configuración geométrica compleja, sometidas a torsión libre.

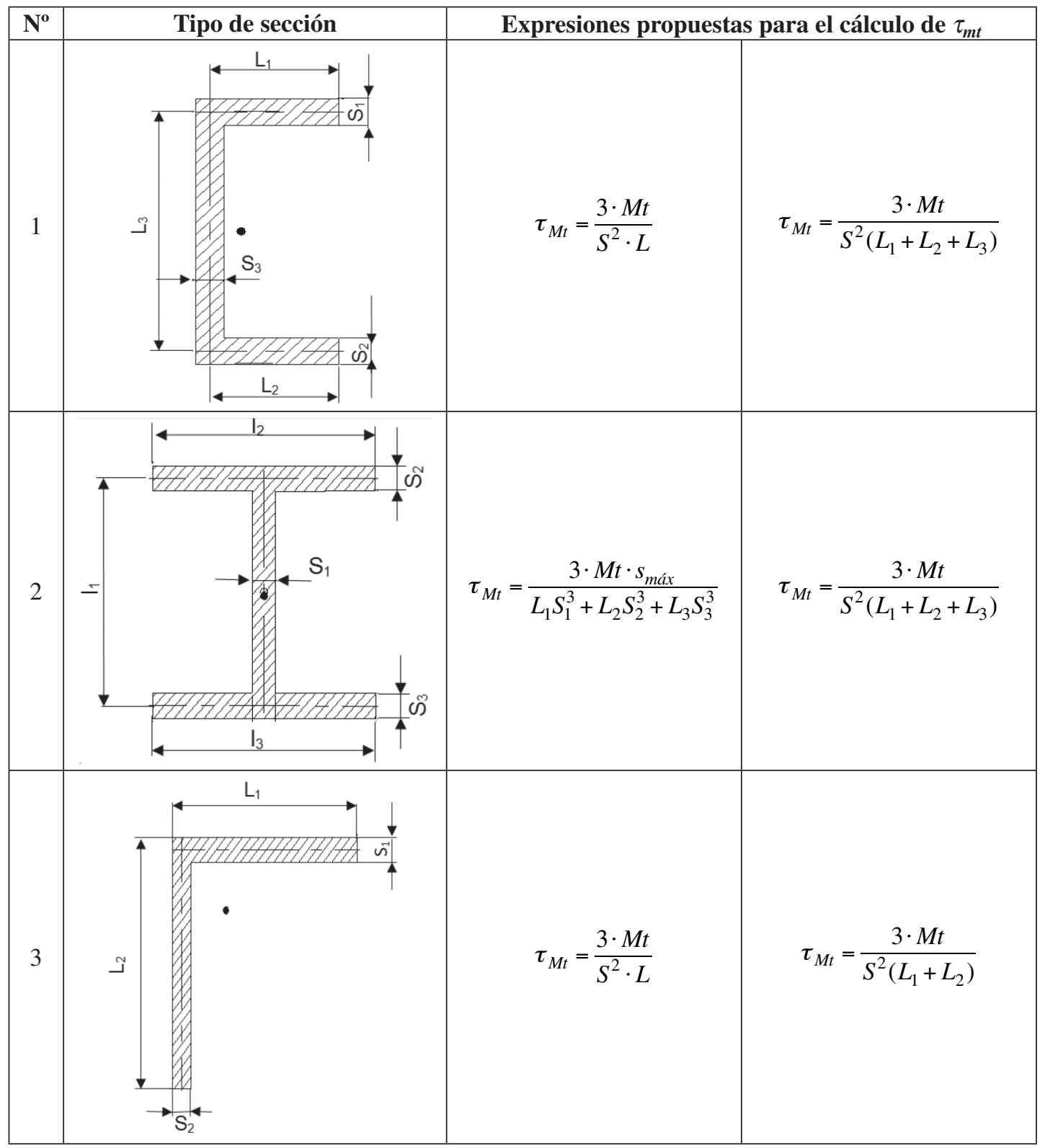


la validez de considerar estas uniones soldadas a tope como perfiles de paredes delgadas. En esta misma Figura 7 se muestra la sección ampliada de la sección inferior de la unión en L señalada, donde se puede apreciar con mayor claridad este planteamiento.

\section{CONCLUSIONES}

Mediante la aplicación de la Teoría de los Perfiles de Paredes Delgadas se obtienen nuevas expresiones para el cálculo de las tensiones tangenciales máximas en tres uniones soldadas de configuración geométrica compleja abierta desarrollable y no desarrollable sometidas a torsión pura.

La modelación por medio del MEF para estas uniones soldadas permitió apreciar claramente que estas se comportan como perfiles de paredes delgadas, ya que los valores de las tensiones máximas se distribuyen uniformemente a lo largo de todo el contorno del perfil siendo mínimas en la línea media de la sección.

Los valores de las tensiones obtenidas analíticamente y por el MEF para cada una de las uniones muestran una excelente correspondencia lo que demuestra la validez de las expresiones propuestas.

\section{REFERENCIAS}

[1] J. Shigley y C. Mischkie. "Diseño en Ingeniería Mecánica”. Editorial Mc Graw Hill Interamericana, p. 943. Ciudad de México, México. 2001.

[2] ANSI/AISC. "Especificación para Construcciones de Acero". No 36010. Asociación Latinoamericana del acero. Santiago, Chile. 2010. ISBN: 978-956-8181-12-3.

[3] A. Hall y H. Lauglhin. "Diseño de Máquinas". Editorial Dossat, p. 344. Madrid, España. 1971.

[4] D. Reshetov. "Elementos de Máquinas". Editorial Pueblo y Educación, p. 830. La Habana, Cuba. 1985.

[5] V.Faires. "Diseño de Elementos de Máquinas". Editorial UTEHA, p. 802. Ciudad de México, México. 1985.

[6] K. Mukanov. "Design of Metal Structures". Editorial MIR, p. 517. Moscú, Rusia. 1968.
[7] P. Schimpke, H.A. Horn y J. Ruge. "Tratado General de Soldadura". Editorial Pueblo y Educación, p. 394. Ciudad de La Habana, Cuba. 1980.

[8] H. Hernández Herrera. "Desarrollo y perfeccionamiento de las expresiones para el cálculo de las tensiones máximas en las uniones soldadas con costuras de filete". Tesis para optar al grado de doctor. Facultad de Mecánica. Universidad de Cienfuegos, p. 90. 2006.

[9] L. Durán, Y. Aguilar, O. Hernández, V. Prado y F. Casanova. "Efecto de la geometría de los depósitos de soldadura de recubrimiento en el desgaste de acero bajo al carbono en contacto con bagazo de caña de azúcar". Ingeniería y Universidad. Vol. $17 \mathrm{~N}^{\circ} 1$, pp. 127-141. 2013. ISSN: 0123-2126.

[10] E. Martínez, V. Eguin, C. Costa y C. Godínez. "Análisis de la penetración en la soldadura de una estructura metálica". Dyna. Vol. 87 $\mathrm{N}^{\circ}$ 1, pp. 90-97. 2012. ISSN: 0012-7353.

[11] E. Díaz, F. Ramos, A. Cruz, M. Rodríguez, J. Pozo, M. Tello y J. Chaparro. "Estudio de la modificación de la composición química en depósitos de soldadura GMAW, empleando diferentes proporciones en la mezcla $\mathrm{CO}_{2}$ $+\mathrm{O}_{2}$ como gas de protección". Ingeniería Mecánica. Vol. $8 \mathrm{~N}^{\circ}$ 2, pp. 67-72. 2005. ISSN: 1815-5944.

[12] E. Martínez, M. Estrems, M. Eguía, A. Garrido y J. Guillén. "Soldadura T.I.G. de acero inoxidable, modelo numérico para el cálculo de temperaturas en la Z.A.T"'. Dyna. Vol. 85 $\mathrm{N}^{\circ}$ 2, pp. 123-130.2010.ISSN:0012-7353.

[13] E. Martínez y M. Estrems. "Desarrollo de un modelo matemático de diferencias finitas para el análisis del campo de temperaturas en la soldadura por arco de chapas finas de acero inoxidable". Revista de Metalurgia. Vol. $46 \mathrm{~N}^{\circ} 6$, pp. 511-519. 2010. ISSN: 0034-8570.

[14] T. Kwang, J. Soo and Y. Chang. "Through Wall welding residual stress profiles for dissimilar metalnozzle butt welds in pressurized water reactors". Fatigue \& Fracture of Engineering Materials \& Structures. Vol. 34, Issue 8, pp. 624-641. 2011. ISSN: 0013-7944.

[15] X. Cao, B. Rivaux, M. Jahazi, J. Cuddy and A. Birur. "Effect of pre- and post-weld heat treatment on metallurgical and tensile 
properties of Inconel 718 alloy butt joints welded using $4 \mathrm{~kW}$ Nd: YAG laser". Journal of material science. Vol. 44, Issue 7, pp. 45574571. 2009. ISSN: 0022-2461.

[16] H. Mansouri and A. Monshi. "Microstructure and residual stress variations in weld zone of flash-butt welded railroads". Science and Technology of Welding and Joining. Vol. 9 $\mathrm{N}^{\circ}$ 3, pp. 237-245. 2004. ISSN: 1362-1718.

[17] D. Stamenković and I. Vasovic. "Finite Element Analysis of Residual Stress in Butt Welding Two Similar Plates". Scientific Technical Review. Vol. 59, Issue 1, pp. 51-60. 2009. ISSN: 2249-6645.

[18] S. González, D. Fernández, J. Álvarez y R. Ramos. "Estudio a fatiga de uniones soldadas a tope. Comparativa y validación de las principales metodologías". Dyna. Vol. 88 $\mathrm{N}^{\circ}$ 2, pp. 171-180. 2013. ISSN: 0012-7353.

[19] O. Araque y N. Arzola. "Estado del arte sobre la integridad estructural de uniones soldadas y modelos de propagación de grietas para la gestión de vida en estructuras". Revista Chilena de Ingeniería. Vol. $21 \mathrm{~N}^{\circ} 2$, pp. 279292. 2013. ISSN: 0718-3305.

[20] A. Moltasov. "Application of the method of nonplanar sections to determine stresses in the zones of concentration caused by weld reinforcement in butt-welded joints". Strength of Materials. Vol. 45, Issue 1, pp. 116-123. 2013. ISSN: 0039-2316.

[21] M. Al-Mukhtar, S. Henkel and H. Biermann. "A Finite Element Calculation of Stress Intensity Factors of Cruciform and Butt Welded Joints for Some Geometrical Parameters". Jordan Journal of Mechanical and Industrial Engineering. Vol. 3, Issue 4, pp. 236-245. 2009. ISSN: 1995-6665.

[22] D. Fernández. "Optimización del diseño a fatiga de uniones soldadas a tope y su aplicación al dimensionamiento de soldaduras transversales de torres eólicas". Tesis para optar al grado de doctor. Departamento de Ciencia e Ingeniería del Terreno y de los Materiales, Universidad de Cantabria. 2012.

[23] P. Urbuan. "Construcción de estructuras metálicas". Editorial Club Universitario. $4^{\text {ta }}$ edición, p. 48. 2010. ISBN: 978-84-8454-913-0.

[24] F. Fraile. "Diseño, Cálculo y Comprobación de Unión Soldada en viga puente de grúa de alma doble". Tesis para optar al grado de magíster. Departamento de materiales. Universidad Politécnica de Madrid. Madrid, España. 2010. 